whilst the yield from patients with a family history of SADS/ SUD or simply abnormal ECG in the absence of symptoms is low. Our overall positivity rate was $20 \%$ with no difference in positivity between the rapid and slow protocols. Both protocols have proven safe to date in our centre, with no significant dysrhythmias or conduction disease identified to date with either protocol. Our incidence of drug induced jaundice was 1 in 207 patients which is higher than previously published and both occurred with the slow protocol. Our data suggests that the rapid protocol is safe and does not result in an increased positivity rate, therefore we will continue with rapid protocol for efficiency in our unit.

\section{LONG-TERM SAFETY OUTCOMES OF PATIENTS IMPLANTED WITH SUBCUTANEOUS IMPLANTABLE CARDIOVERTER DEFIBRILLATORS}

N Starr, S Esmonde, N McKeag, E Chew. Mater Misericordiae University Hospital, Dublin, Ireland

\subsection{6/heartjnl-2021-ICS.13}

Introduction Boston Scientific (location) Emblem ${ }^{\mathrm{TM}}$ subcutaneous implantable cardioverter defibrillator (SICD) is an extrathoracic defibrillator system. Two landmark studies, S-ICD System IDE Clinical Investigation (IDE) and Boston Scientific Post Market S-ICD Registry (Effortless) demonstrated safety profiles similar to transvenous (TV) implantable cardioverter defibrillators (ICDs). Aim: This study aimed to investigate the working safety profile of Boston Scientific SICD Emblem ${ }^{\mathrm{TM}}$ devices implanted in patients attending a hospital trust within the United Kingdom (UK) between February 2015 and 1st March 2020.

Methods This was a single-center, retrospective study that investigated patients followed up at a single UK hospital trust. Data was collected using Boston Scientific Latitude ${ }^{\mathrm{TM}}$ NXT and patient electronic care records.

Results A total of 116 patients with a Boston Scientific Emblem $^{\text {TM }}$ SICD were included in the study. 115 patients had the SICD implanted at the Hospital Trust whilst 1 patient had their implant completed at an external paediatric center. Patient characteristics including SICD indication are summarized in table 1 . Over the course of the study follow-up 20 patients experienced at least one event, which were subsequently stratified into appropriate or inappropriate events. 7 (6\%) patients received an appropriate shock for ventricular arrhythmias whilst 7 (6\%) also received an inappropriate shock (IAS). Patients that received inappropriate therapies had a total of 12 shocks delivered overall. Of the total shocks delivered, 7 (58\%) were secondary to non-physiological noise believed to be myopotential oversensing. Of note there were no IAS due to T-wave oversensing. A summary of observed outcomes is presented in Table 2. The number of reinterventions and their indications are detailed in figure 2. A total of $3(2.6 \%)$ patients died during the study follow-up but none of the deaths were directly attributable to the device or implant procedure. The mean age at death was $67 \pm 13 \mathrm{yrs}$, with a mean duration from implant to death of $12.6 \pm 10.8$ months.

Conclusion This study confirms an ongoing working safety profile of implanted SICDs consistent with previous SICD

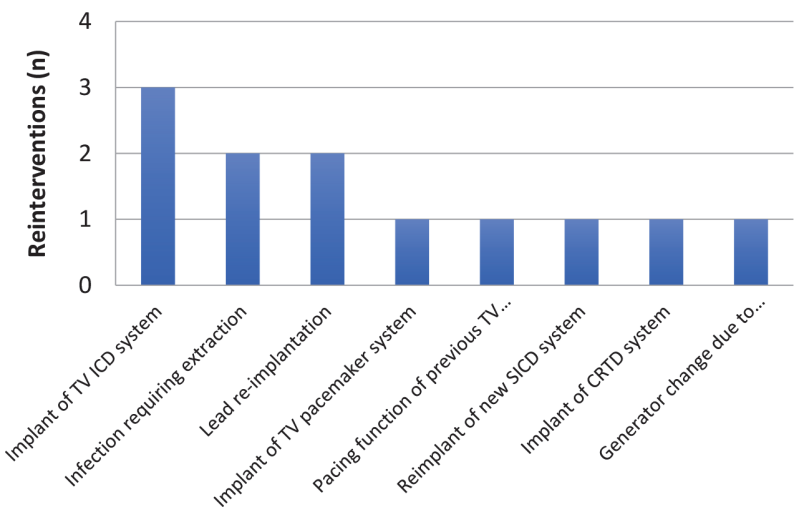

Abstract 13 Figure 2 Breakdown of Reinterventions: TV transvenous, ICD - implantable cardioverter defibrillator, SICD subcutaneous implantable cardioverter defibrillator, CRTD - cardiac resynchronisation therapy defibrillator

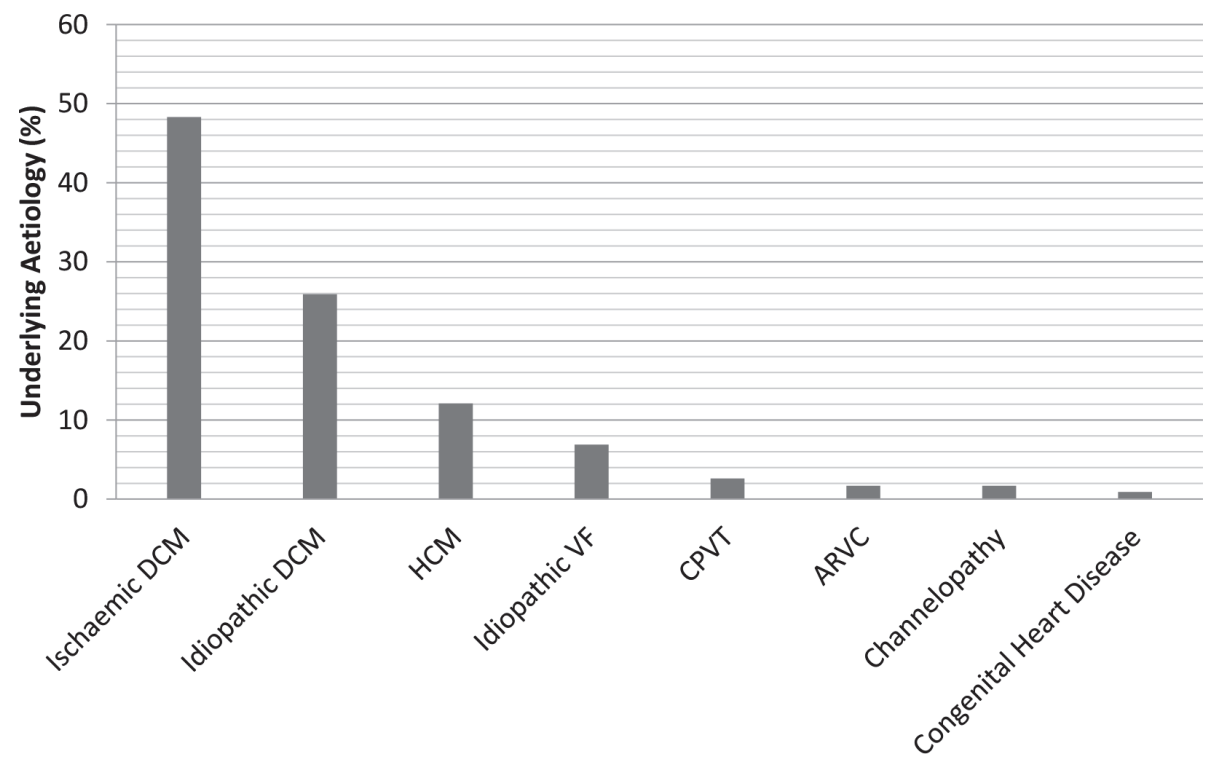

Abstract 13 Figure 1 Underlying Aetiology DCM-dilated cardiomyopathy, HCM-hypertrophic cardiomyopathy, ARVC arrhythmogenic right ventricular cardiomyopathy, VF-ventricular fibrillation, CPVT-catecholaminergic polymorphic ventricular tachycardia 
Abstract 13 Table 1 LVEF-left ventricular ejection fraction, DCMdilated cardiomyopathy, HCM-hypertrophic cardiomyopathy, ARVC arrhythmogenic right ventricular cardiomyopathy, VF-ventricular fibrillation, CPVT-catecholaminergic polymorphic ventricular tachycardia, AF -Atrial fibrillation

\begin{tabular}{ll}
\hline & All patients $(\mathbf{n}=116)$ \\
\hline Total & 116 \\
Age at implant [mean years] & $51 \pm 14$ years \\
Male [n (\%)] & $86(68)$ \\
Female [n (\%)] & $30(32)$ \\
LVEF [mean\%] & 36.7 \\
Primary prevention [n (\%)] & 81 \\
Secondary prevention [n (\%)] & 35 \\
AF [n (\%)] & $28(24)$ \\
Follow-up post device implantation [mean months] & $24.3 \pm 15.9$ months (range 1.8 - 62.9) \\
\hline
\end{tabular}

Abstract 13 Table 2 SVT supraventricular tachycardia

\begin{tabular}{|c|c|c|c|}
\hline & Result: & & Result: \\
\hline Number of Total events & 44 & $\begin{array}{l}\text { Number of incorrectly labeled } \\
\text { ventricular events (no therapy } \\
\text { delivered) }\end{array}$ & 12 \\
\hline $\begin{array}{l}\text { Number of patients } \\
\text { (\% of total) }\end{array}$ & $\begin{array}{l}22 \\
(18.9)\end{array}$ & Number of patients ( $\%$ of total) & 7 (6) \\
\hline Appropriate shocks: & & $\begin{array}{l}\text { T wave oversensing (event number with } \\
\text { no therapy delivered) }\end{array}$ & 1 \\
\hline Number of events & 15 & $\begin{array}{l}\text { Non cardiac oversensing (event number } \\
\text { with no therapy delivered) }\end{array}$ & 11 \\
\hline $\begin{array}{l}\text { Number of patients } \\
\text { (\% of total) }\end{array}$ & 7 (6) & $\begin{array}{l}\text { SVT (event number with no therapy } \\
\text { delivered) }\end{array}$ & 0 \\
\hline \multicolumn{4}{|l|}{ Inappropriate shocks: } \\
\hline Number of events & 12 & Reintervention: & \\
\hline \multirow[t]{2}{*}{$\begin{array}{l}\text { Number of patients } \\
\text { (\% of total) }\end{array}$} & 7 (6) & Number of procedures & 12 \\
\hline & & Number of patients ( $\%$ of total) & $9(7.8)$ \\
\hline
\end{tabular}

studies. This study however highlights real time data offering further insight into potential issues associated with SICDs. One of the main findings from the study was the fact that of the $6 \%$ of patients that received an IAS $58 \%$ were deemed due to myopotential oversensing. None of the inappropriate shocks detected were due to $\mathrm{T}$ wave oversensing, an issue that appears to have been resolved with the Emblem $^{\mathrm{TM}}$ SMART pass filter. IAS due to myopotential oversensing is therefore an important practical outcome from the study. The impact of myopotential oversensing suggests lead position in relation to the pectoral muscle poses real difficulties and should be taken into consideration as early as vector analysis prior to implant. The importance of detecting non-physiological noise was highlighted by an advisory notification released in December 2020. The advisory highlighted a lead fracture risk at the region of the proximal sensing probe in certain electrodes and generally requires replacement. To detect both non-physiological noise and myopotential interference remote surveillance is therefore an important aspect of care in patients with SICD implants.

\section{THE DA VINCI STUDY: DYSLIPIDAEMIA MANAGEMENT IN IRELAND AND EUROPE}

${ }^{1} \mathrm{G}$ Offiah, ${ }^{1} \mathrm{C}$ O'Connor, ${ }^{2} \mathrm{C}$ Kennedy, ${ }^{3} \mathrm{~J}$ Gallagher, ${ }^{2,4} \mathrm{P}$ O'Connor, ${ }^{5,6} \mathrm{BF}$ McAdam, ${ }^{7} \mathrm{KK}$ Ray, ${ }^{8} \mathrm{M}$ Schoonen, ${ }^{1,4,9} \mathrm{~V}$ Maher. ${ }^{1}$ Department of Cardiology, Tallaght University Hospital, Dublin, Ireland; '2Department of Pharmacology \& Therapeutics, St James's Hospital, Dublin, Ireland; ${ }^{3}$ The Palms GP Surgery, The Avenue, Gorey, Co Wexford, Ireland; ${ }^{4}$ Trinity College, Dublin, Ireland; ${ }^{5}$ Department of Cardiology, Beaumont Hospital, Dublin, Ireland; ${ }^{6}$ Royal College of Surgeons, Dublin, Ireland; ${ }^{7}$ mperial Centre for Cardiovascular Disease Prevention and Imperial Clinical Trials Unit, Imperial College London, London, UK; ${ }^{8}$ Amgen Centre for Observational Research, Uxbridge, UK; ${ }^{9}$ Advanced Lipid Management and Research ALMAR Centre, Tallaght University Hospital, Dublin, Ireland

\subsection{6/heartjnl-2021-ICS.14}

Introduction Elevated low-density lipoprotein cholesterol (LDLc) is a major risk factor for atherosclerotic cardiovascular (CV) disease. Robust evidence has shown that lowering LDLc levels results in reduced incidence of CV events. For every $1 \mathrm{mmol} / \mathrm{L}$ reduction in LDLc, there is a $22 \%$ reduction in CV events. Consequently, the 2019 European Society of Cardiology/European Atherosclerosis Society (ESC/EAS) dyslipidaemia guidelines are advocating lower LDLc goals than the 2016 guidelines. Achieving lipid goals is more challenging at a population level. Our study aims to evaluate lipid goal achievement in Ireland compared to its European counterparts.

Methods The DA VINCI study is a cross-sectional observational study that took place between June 2017 and November 2018, across 18 European countries, to assess lipid goal achievement. The 2016 ESC dyslipidaemia guidelines were in use during the study period when the data were collected. A total of 5888 patients were recruited. The subgroup analysis presented here characterises the findings in primary and secondary prevention patients from Ireland $(n=198)$. Data were collected on background history, lipid modifying therapy (LMT) and LDLc at the time of enrolment. Continuous variables were reported as means, standard deviations, medians, interquartile range with $\mathrm{min} / \mathrm{max}$ values. Categorical data were analysed using chi-square test.

Results High intensity statin use was more prevalent in the Irish cohort than the overall European cohort $(43.9 \%$ vs $34.4 \% ; p=0.006)$. There were $12.6 \%$ of Irish patients prescribed ezetimibe, with only $6 \%$ treated with other forms of non-statin LMT. There was no significant difference in the use of non-statin LMT between the Irish and European groups. Overall, LDLc goal achievement was suboptimal in Ireland and Europe, however, the Irish cohort was more likely to attain lipid goals according to the 2016 guideline goals (64.1\% vs $53.9 \%, p=0.014$ (figure 1). Fewer patients overall met the lower 2019 LDLc goals, but there was a non-significant trend towards more patients achieving goals in Ireland (40.5\% vs $33.5 \%, \mathrm{p}=0.06)$. Mean LDLc $(\mathrm{mmol} / \mathrm{L})$ was similar in Irish patients stabilised on LMT (2.14 vs 2.37) and those that were treatment naïve (3.50 vs 3.61).

Conclusion/Implications LDLc goal achievement is suboptimal, although in this study, Ireland performed better than the overall European cohort in guideline directed lipid goal attainment. The use of high intensity statins was limited but more common in the Irish cohort; however, it is clear that combination treatment was underutilised. Given the mortality benefit associated with profound reductions in LDLc, a more aggressive approach to 2019 ESC/EAS guideline implementation is 